\title{
La querella de las mujeres en Castilla (siglo XV) y su relación con la historia de las mujeres y la historia de género
}

\section{The "querelle des femmes" in Castile $\left(15^{\text {th }}\right.$ century) and its relation to women's history and gender history}

\author{
Roque Sampedro \\ Universidad de Santiago de Compostela \\ roquesampedro@gmail.com
}

\section{Resumen}

Este artículo pretende explorar las conexiones y particularidades de la historia de las mujeres y la historia de género a través de un ejemplo medieval: la llamada querella de las mujeres en la Castilla del siglo XV. Primero, se describirán brevemente los rasgos fundamentales de este debate sobre la condición femenina. A continuación, se analizará brevemente el surgimiento de la historia de las mujeres y la interpretación que se hace de la querella de las mujeres dentro de esta corriente historiográfica. En tercer lugar, se estudiarán la aplicación de postulados del feminismo de la diferencia a la querella en el ámbito castellano. Finalmente, se explicará la emergencia de la historia de género y se expondrán algunas de las posibles aproximaciones desde esta perspectiva al análisis de la querella de las mujeres.

\section{Palabras clave}

Historia de las mujeres, historia de género, querella de las mujeres, feminismo de la diferencia

\begin{abstract}
This article aims to explore the connections and particularities of women's history and gender history through a medieval example: the so-called "querelle des femmes" in fifteenth century Castile. First, the fundamental traits of this debate about the feminine condition will be described. Next, I will analyze the emergence of women's history and the interpretation of the "querelle des femmes" within this historiographical tendency. In the third place, the use of the postulates of difference feminism in relation to the debate in a Castilian context will be studied. Finally, the emergence of gender history will be explained, and some of the posible approaches to the analysis of the "querelle des femmes" from this perspective will be layed out.
\end{abstract}

\section{Key Words}

women's history, gender history, querelle des femmes, difference feminism 


\section{Introducción}

Este texto surge de la posibilidad de explorar las relaciones historiográficas entre la historia de las mujeres y la historia de género, partiendo de la querella de las mujeres. Se trata de un debate sobre la condición femenina que se extiende desde el siglo XV al XVIII, aunque nos centraremos en sus manifestaciones en la Castilla del siglo XV. Así, nos plantearemos cómo se vinculan teoría -los elementos conceptuales de la historia de las mujeres y la historia de género-con práctica -la querella de las mujeres como objeto de estudio-. El objetivo es, entonces, poner en contexto el estudio de la querella dentro del marco global de estas dos líneas historiográficas, y delinear, brevemente, algunas de las ventajas de una aproximación desde la historia de género. Se trata de realizar un aclarado de ideas, tanto teóricas como prácticas, en relación a la historia de las mujeres, a la historia de género y a la querella de las mujeres. Por una parte, nos hemos basado en las reflexiones en torno al desarrollo de la historia de las mujeres y la historia de género de un conjunto de historiadoras implicadas en estas prácticas historiográficas (Joan Scott, Gissela Bock, Joan Kelly, Michelle Perrot o Isabel Morant, por ejemplo), lo que nos ha permitido profundizar en los marcos teóricos específicos de cada fase de desarrollo de estas corrientes, así como poner esta evolución en relación con sus contextos históricos - principalmente, la emergencia del feminismo y los estudios de género-. Por otra parte, a la hora de tratar la querella hemos recurrido principalmente al trabajo historiográfico a partir de una serie de ejemplos que tratan tanto algunos de sus elementos generales -el papel de Christine de Pizan-, como abordan aspectos de la querella específicos del caso castellano, con las intervenciones de Alfonso Martínez de Toledo, Juan Rodríguez del Padrón, Diego de Valera, Álvaro de Luna y Teresa de Cartagena.

Así, en este artículo se procederá, en primer lugar, a trazar los rasgos generales de la querella de las mujeres en el siglo XV, identificando autores, textos, geografías, ideas y contextos sociales; centrándonos en el caso castellano e indicando cómo se pueden entender las defensas de mujeres como representaciones propias de un grupo social determinado que coincidiría, a grandes rasgos, con los círculos cortesanos durante el reinado de Juan II. En segundo lugar, se describirá la emergencia de la historia de las mujeres en los años 60 y 70, y sus preocupaciones principales (narrar una historia de la agencia femenina, estudiar las implicaciones de esta historia) y veremos, a través de Joan Kelly, cómo estas preocupaciones se plasman en el estudio de la querella. En tercer lugar, se analizarán los rasgos generales de la historia de las mujeres en los años 80, en relación a nociones como "cultura femenina". Se estudiará cómo conceptos de carácter similar influyen a un grupo de historiadoras españolas que han interpretado la querella dentro del marco teórico del feminismo de la diferencia, comentando lo que nos parece que son algunos problemas de sus análisis. Finalmente, en cuarto lugar, veremos cómo la crítica de ciertos aspectos de la historia de las mujeres da lugar, en los años 90, a la historia de género que, aunque comparte algunos objetos en común, es una forma de hacer historia diferenciada. Además, intentaremos utilizar conceptos propios de la historia de género para interpretar algunos aspectos de la querella de las mujeres en el caso castellano, como la importancia de la relación entre las diferencias de género y otras diferencias sociales y el estudio de las masculinidades. 


\section{1. ¿Qué es la querella de las mujeres en la Edad Media?}

El estudio de la querella de las mujeres refleja bien las preocupaciones de la historia de las mujeres -la agencia y conciencia femeninas, las categorías de análisis, o la periodización- y la historia de género -el estudio de las relaciones entre los sexos en conjunción con otras diferencias sociales o el estudio de las masculinidades-. Definamos primero este fenómeno. La querella de las mujeres se entiende como un debate o conjunto de debates en torno a la cuestión de la "inferioridad natural" de la mujeres y la "superioridad natural" de los hombres. Estos debates no se sitúan únicamente en el siglo $\mathrm{XV}$, sino que se han ubicado cronológicamente desde la Baja Edad Media hasta el siglo XVIII e implican diversas disciplinas (filosofía, teología, medicina, política y literatura). La caracterización de Margarete Zimmermann del fenómeno, por ejemplo, destaca su complejidad: "le combat est engagé autour d'un imaginaire, d'un réseau de concepts et d'images, d'un espace conceptuel se rapportant aux notions de 'féminin/masculin' et à leur construction par les 'créateurs' d'images et de textes". ${ }^{1}$ Es posible describir más detalladamente algunas de las características de la querella, especialmente para el siglo XV.

Los comienzos de la querella suelen situarse en Francia, con la intervención de Christine de Pizan (1364-1430), que escribe entre 1404 y 1405, La ciudad de las damas, como una defensa ante las diatribas anti-femeninas de, por ejemplo, el Roman de la Rose. Cabe destacar la vinculación de esta autora con la corte de Carlos VI e Isabel de Baviera. ${ }^{2}$ Un segundo espacio sería la corte de Juan II de Castilla (1406-1454), donde la querella estalla como respuesta al Corbacho de Alfonso Martínez de Toledo, muy crítica con el sexo femenino. Así, las intervenciones principales serían las de Juan Rodríguez del Padrón, Triunfo de las donas (1444), ${ }^{3}$ de Diego de Valera, Tratado en defensa de virtuosas mugeres $(1445),{ }^{4}$ y de Álvaro de Luna, Libro de claras y virtuosas mugeres (1446). ${ }^{5}$ Hay que destacar también la obra posterior de Teresa de Cartagena, Admiraçión operum Dey (segunda mitad el siglo XV), escrita como respuesta a las acusaciones de plagio relativas a una obra anterior. ${ }^{6}$ Un último espacio sería la Península Itálica, en la que se encuentran la obra de Nicolosa Castellani Sanuti, Para que les sea restituido el ornato a las mujeres, de 1453, que critica la prohibición de ornamentos y vestidos suntuarios en Bolonia; y en Brescia, el caso de Laura Cereta,

\footnotetext{
${ }^{1}$ Margarete Zimmermann, "Querelle des femmes, querelles du livre", en Dominique de Courcelles y Carmen Val Julián, Des femmes et des livres. France et Espagnes, XIV-XVIIe siècle, Actes de la journée d'étude organisée par l'École nationale des chartes et l'École normale supérieure de Fontenay/SaintCloud, Paris, 20 avril 1998 (École des chartes : Paris, 1999), 87.

2 Ana Vargas Martínez, "La ciudad de las damas' de Christine de Pizan: obra clave de la querella de las mujeres', en Cristina Segura Graíño (coord.), La querella de las mujeres, vol. 1: Análisis de textos (A.C. Al-Mudayna: Madrid, 2009), 21-27.

3 Ana Vargas Martínez, "Sobre los discursos políticos a favor de las mujeres (El Triunfo de las donas de Juan Rodríguez de la Cámara)", Arenal, vol. 20, 2 (2013): 263-288.

${ }^{4}$ Florence Serrano, "Del debate a la propaganda política mediante la querella de las mujeres en Juan Rodríguez del Padrón, Diego de Valera, y Álvaro de Luna”, Talia dixit: revista interdisciplinar de retórica e historiografía, 7 (2012): 103-106.

${ }^{5}$ María Jesús Fuentes Pérez, "Voces profemeninas en la querella de las mujeres: Álvaro de Luna y El libro de las claras y virtuosas mujeres”, en C. Segura Graiño, La querella, 105-129.

${ }^{6}$ María Milagros Rivera Garretas, "La Admiración de las obras de Dios de Teresa de Cartagena y la querella de las Mujeres”, en Cristina Segura Graíño, La voz del silencio, vol. 1: Fuentes directas para la historia de las mujeres (A.C. Al-Mudayna: Madrid, 1992), 277-300.
} 
quien en varias epístolas redactadas entre 1488 y 1492, se defiende de acusaciones de plagio hacia su obra de astronomía.

Estos casos presentan una serie de características comunes, entre las que es conveniente destacar:

a) En primer lugar, el propio período. El siglo XV se presenta como una época en la que se multiplican los textos sobre la condición de la mujer. ${ }^{7}$

b) Por otra parte es necesario destacar el carácter polémico de los textos. Nos referimos a que las intervenciones en la querella se caracterizan o bien por ser respuestas a otros textos, o estar dirigidas a otros textos. Christine de Pizan afirma haber escrito la Ciudad de las damas para refutar las posiciones "misóginas" medievales ${ }^{8}$ de textos como el Roman de la Rose de Jean de Meun o las Lamentaciones de Mateolo.

c) Finalmente, al menos en los casos de Francia y Castilla, la importancia de las cortes como un espacio político y cultural en torno al cual se producen las intervenciones en la querella. Este aspecto es relevante ya que, como se argumentará, los grupos sociales que podemos relacionar con las representaciones de la querella están posiblemente relacionados con la sociedad cortesana.

A estos elementos habría que incluir un conjunto de cuestiones que integran varios de los discursos que forman parte de las intervenciones a favor de las mujeres:

a) En primer lugar, la idea de que las diferencias entre hombres y mujeres, en algunos aspectos, vienen dadas por una educación diferente. ${ }^{10}$ Álvaro de Luna, por ejemplo, afirma que las "menguas" de las mujeres son producto de la costumbre, no de la naturaleza. ${ }^{11}$ Christine de Pizan insiste además en la necesidad de educación de la mujer, algo seguramente relacionado con la influencia humanista, que permitía el acceso al estudio de la gramática y la literatura para las mujeres. ${ }^{12}$

b) Otro elemento relevante es la defensa y admiración de las virtudes consideradas

\footnotetext{
${ }^{7}$ Monserrat Cabré i Pairet, "Estrategias de des/autorización femenina en la querella de las mujeres, s. XV”, en Cristina Segura Graíño (coord.), La educación de las mujeres ¿subordinación o libertad? (A.C. Al-Mudayna: Madrid,1996), 77-90.

${ }^{8} \mathrm{Se}$ ha atribuido un carácter misógino al pensamiento medieval sobre las mujeres y en especial de los autores "críticos" con las mujeres dentro del marco de la querella. En relación al concepto de misoginia medieval, Howard Bloch la define como un acto del habla en el cual la mujer es el sujeto de una oración y el predicado de un término general, o cuando se emplea el sustantivo mujer con una "M" mayúscula. Es decir, como cualquier generalización que asigne una característica a todas las mujeres por igual, ya sea positiva o negativa. Sin embargo, esta definición supone, al menos en el ámbito de la querella, clasificar una gran cantidad de las intervenciones, ya sea a favor o en contra de las mujeres, como misóginas, lo que hace perder valor analítico al concepto, al menos, para el estudio de esta controversia. Véase R. Howard Bloch, Medieval Misoginy and the Invention of Western Romantic Love (The University of Chicago Press: Chicago \& London, 1991), 5-6.

9 A. Vargas Martínez, "La ciudad de las damas"”, 21-32.

${ }^{10}$ Joan Kelly, "Early feminist theory and the 'Querelle des femmes', 1400-1789”, Signs, vol. 8, 1 (1982): 5-7.

${ }^{11}$ M. J. Fuentes Pérez, "Voces profemeninas", 109-111.

${ }^{12}$ A. Vargas Martínez, "'La ciudad de las damas"”, 34-36.
} 
femeninas, como la castidad, la piedad, la obediencia o la humildad.

c) Parte de la defensa de las mujeres se hace a partir de relatos de mujeres históricas o mitológicas, que sirven como modelo de mujer virtuosa, algo presente en prácticamente todas las obras. La idea es defender las capacidades para la virtud de la mujer a través de casos conocidos de mujeres sobresalientes: diosas de la Antigüedad Clásica, mujeres bíblicas o santas.

d) En último lugar, cabe destacar la inversión de la interpretación tradicional del Génesis, en la cual Eva acarrea las culpas de Caída, falta que se extiende a todas las mujeres. Siendo este uno de los puntos críticos en las "difamaciones" de las mujeres, algunos textos en defensa de las mujeres dedican un espacio a la puesta en valor de la figura de Eva.

Todos estos símbolos, relatos, ideas o explicaciones relativos a la condición femenina son representaciones colectivas que podemos pensar como propias de un grupo social determinado. Este grupo, además, no sería un grupo marcado por un único rasgo social (sexo, religión, clase socioeconómica, etc.) sino que se trataría de colectivos compuestos, como instituciones, sectores sociales amplios o, en definitiva, comunidades que están cohesionadas, al menos parcialmente, por la existencia de estas representaciones. ${ }^{13}$ Así, es posible plantearse que las defensas de las mujeres reflejan la conciencia de un determinado grupo social, por lo menos en el caso castellano.

Con esta idea en mente, podemos situar la querella de las mujeres en la corte de Juan II (1406-1454), lo que obedece a varios motivos. Se trata de un espacio y un momento de notable actividad cultural, caracterizado por una relación intensa con el humanismo italiano. La corte funciona como un centro de poder en la Baja Edad Media, con lo que esto conlleva en la producción de representaciones tanto legitimadoras como normativas. ${ }^{14}$ Por otra parte, es un espacio con el que están relacionados varios de los participantes en la querella, desde el Arcipreste de Talavera (capellán del rey), Álvaro de Luna, Diego de Valera y Juan Rodríguez de Padrón. Estos dos últimos, además, dedican sus textos a la reina María de Aragón, un personaje activo culturalmente, que promueve la composición y traducción de textos. ${ }^{15}$ La reina no es la única que promociona la composición de defensas. Admiraçión operum dey, de Teresa de Cartagena, que funciona en parte como una defensa de mujeres, fue escrita a petición de Juana de Mendoza, una noble perteneciente a la sociedad cortesana, así como su marido Gómez de Manrique. En este sentido, nos parece que el grupo social del que emergen las representaciones de la querella de las mujeres, coincide en buena medida con la sociedad cortesana. Así, la defensa de un ideal de mujer noble y virtuosa viene a legitimar a las mujeres de la Corte, siempre que estas cumplan con dichas condiciones.

\footnotetext{
${ }^{13}$ Sacamos esta idea de Roger Chartier, El mundo como representación. Historia cultural: entre práctica representación (Gedisa: Barcelona, 1999), 27-28, 48-50.

${ }^{14}$ Hervé Martin, Mentalités Médiévales: XIe-XVe siècle (Presses Universitaires de France: Paris, 1996), 11-14; y Jacques Le Goff, Les mentalités: une histoire ambigüe' en Jacques Le Goff y Pierre Nora (dirs.), Faire l'histoire, vol. 3: nouveaux objets, Gallimard, París, 1974, pp. 85-88.

${ }^{15}$ Ana Vargas Martínez, "Lo que está vivo puede llegarnos. Una lectura desde la diferencia sexual de los tratados escritos por hombres en favor de las mujeres (Corona de Castilla, siglo XV)", en Montserrat Cabré i Pairet y otros, De dos en dos. Las prácticas de creación y recreación de la vida y la convivencia humanas (Horas y horas: Madrid, 2000), 88-90.
} 
No obstante, es importante destacar que si bien es posible que el grupo social que produce estas representaciones pertenezca a la Corte, esto no implica que todos los miembros de la misma estén incluidos en dicho grupo social. Las nociones e ideales de mujer empleadas en las defensas no tienen por qué representar la forma de pensar de todos los cortesanos. Posiblemente, las representaciones de la controversia excluyen a miembros de la sociedad cortesana -empezando, como veremos, por los propios difamadores- que no podemos identificar aquí.

Así, la querella de las mujeres en el siglo XV tiene una serie de características que la hacen un posible objeto de la historia de las mujeres y la historia de género. Ahora bien, la conceptualización de este fenómeno como objeto historiográfico se hace posible con el desarrollo de diferentes perspectivas teóricas, como el feminismo de la diferencia, dentro del ámbito de la historia de las mujeres. Es desde esta corriente historiográfica desde donde se emplean categorías como patriarcado o des/autorización para entender la querella. Así, podemos comprender el estudio de este debate a partir de los cambios historiográficos que supone la emergencia de la historia de las mujeres.

\section{La querella de las mujeres dentro de la historia de las mujeres}

Se ha destacado la existencia de formas de historiar la vida de las mujeres desde al menos el siglo XIX. ${ }^{16}$ Las mujeres podían aparecer en relatos históricos tradicionales, especialmente mujeres que hubieran destacado por su papel en la vida política, mujeres consideradas excepcionales y por ello eran merecedoras de aparecer en relatos relativos a la guerra o la diplomacia, pero sin apenas tener en cuenta la condición sexuada del ser humano en otros ámbitos. Y aun así, la feminista Virgina Wolf se quejaba en los años 20 de la ausencia de las mujeres en los libros de historia, lo que suponía que las mujeres de su generación no tenían modelos a los que seguir. ${ }^{17}$ En este sentido, sería necesario un cambio conceptual que pusiera sobre la mesa los elementos necesarios para poner en práctica una historia que tuviera en cuenta las diferencias entre los dos sexos, y que en buena medida hace posible el estudio contemporáneo de la querella de las mujeres.

La emergencia de la historia de las mujeres, en los años 70, supone entonces la posibilidad de una interpretación de la historia en términos sexuados, es decir, teniendo en cuenta que las sociedades se componen tanto de mujeres como de hombres, y que existen diferencias sociales entre ambos, en relación al estatus legal, a la actividad económica, a la actividad política o a la posición social. Este cambio viene dado por varios factores que, sin ánimo de ser exhaustivos, describiremos aquí:

a) La obra de Simone de Beauvoir, El segundo sexo (1949), que indaga de forma filosófica en la formación histórica de un "segundo sexo", las mujeres, dependiente de los varones. Se trata de una obra que tras un escándalo inicial, resultaría muy influyente en los movimientos feministas en Francia, a partir de finales de los 60. El texto sienta las bases a la hora de desnaturalizar al menos algunas de las diferencias entre hombres y mujeres, y en resaltar la situación de marginación política, social y económica que las

\footnotetext{
${ }^{16}$ Elena Hernández Sandoica, Tendencias historiográficas actuales. Escribir historia hoy (Akal: Madrid, 2004), 437-439.

17 Carmen Pallares Méndez, Historia das mulleres en Galicia. Idade Media (Nigratrea: Santiago de Compostela, 2011), 15-21.
} 
mujeres han experimentado históricamente. ${ }^{18}$

b) La emergencia, especialmente en Estados Unidos, de la llamada "segunda ola" del feminismo, a partir de los años 60. Mientras que la "primera ola" tenía como meta acabar con los obstáculos legales a los que se enfrentaban las mujeres, teniendo como objetivo principal la obtención del derecho a voto - de ahí la expresión de las "sufragistas"-, la segunda ola presenta una serie de intereses más variados, que abarcan desde la sexualidad al trabajo, preocupaciones que estarán presentes en la historiografía de los inicios de la historia de las mujeres. ${ }^{19}$

c) La formación progresiva de los "estudios de mujeres" -women's studiesconcebidos como espacios interdisciplinares para el estudio de la situación de las mujeres. Es en este espacio académico en el que se desarrolla la teoría feminista, desde diferentes perspectivas disciplinares y teóricas. Es el momento, por ejemplo, de auge en el empleo del término "patriarcado", para designar los sistemas sociales en los que aparece una jerarquía entre varones y mujeres, es decir, dominación masculina y subordinación femenina. Aparece además, cierta variedad a la hora de explicar los orígenes de dicha dominación: puede ser un producto de la transformación del sexo en una categoría política (Kate Millet), o de la extracción de plusvalías al trabajo femenino (Shulamith Firestone). ${ }^{20}$

Si bien todos estos elementos influirán de una manera u otra en el desarrollo de la historia de las mujeres, la preocupación inicial de las historiadoras feministas era, en primer lugar, la de restablecer las mujeres a la historia, y en segundo, preguntarse acerca de las posibilidades de existencia de una historia de las mujeres como un campo de estudio diferenciado dentro de la disciplina. Así, por ejemplo, en 1973, cuando tienen lugar en Francia las primeras actividades académicas que buscan indagar sobre las posibilidades de una historia de las mujeres, Michelle Perrot, Fabienne Bock y Pauline Schmitt-Pantel plantean un seminario que lleva por título "Les femmes ont-elles une histoire?". ${ }^{21}$ El título es bastante indicativo del planteamiento: se trataría de poner en duda, de desarticular la idea de que todas las diferencias entre los sexos son consustanciales al ser humano y su condición sexuada. De esta forma sería posible hacer una historia de, por lo menos, aquellos aspectos que no vienen determinados por un aspecto invariable de la mujer, sino que son producto del contexto social, cultural o político.

\footnotetext{
${ }^{18}$ Isabel Morant, "Historia de las mujeres e historia: innovaciones y confrontaciones", en Carlos Barros (ed.) e Israel Sanmartín (coord.), Pablo Chaves (secr.), Historia a Debate, t. III, Problemas de Historiografía. Actas del II Congreso Internacional "Historia a Debate", celebrado del 14 al 18 de Julio de 1999 en Santiago de Compostela (Historia a Debate: Vedra, 2000), 293.

${ }^{19}$ No cabe ver el feminismo "de segunda ola" como un movimiento unitario. Algunos aspectos, como por ejemplo las diferencias entre feministas liberales, socialistas y radicales, o los debates en torno a los conceptos de igualdad, diferencia o identidad, pueden verse en Yasmine Ergas, "El sujeto mujer: el feminismo de los años sesenta-ochenta”, en G.Duby y M. Perrot, Historia de las mujeres en Occidente. El siglo XX (Taurus: Barcelona, 1991), 593-620.

${ }^{20}$ Anna Fernández Poncela, "Estudios sobre las mujeres, el género y el feminismo", Nueva Antropología. Revista de Ciencias Sociales (1998): 80.

${ }^{21}$ Michelle Zancarini-Fournel, "Histoire des femmes, histoire du genre", en Christian Delacroix, François Dosse, Patrick García y Nicolas Offenstadt (dirs.), Historiographies, I. Concepts et débats (Gallimard: París, 2010, 208.
} 
La preocupación de la historia de las mujeres, en un primer momento es, entonces, encuadrar a las mujeres dentro del discurso historiográfico. Es el momento de desarrollo de lo que se llamó, en el mundo anglo sajón, "her-story", es decir, una historia centrada exclusivamente en la agencia y experiencia femenina, y su participación en los procesos históricos. $^{22}$ Además, la importancia de la historia económica y social hace que las preocupaciones sobre la experiencia femenina se plasmen principalmente dentro de estos ámbitos. Las mujeres se incluyen dentro de la historia del trabajo o del movimiento obrero, o el estudiar el desarrollo demográfico diferenciando entre hombres y mujeres, así como el estudio de la familia, que sería un campo fructífero de estudio. ${ }^{23}$ En España, por ejemplo, donde el desarrollo de la historia de las mujeres ha sido más tardío, los primeros trabajos colectivos referidos encuadrados en el mundo medieval se refieren, precisamente, al marco jurídico de las mujeres y al mundo del trabajo de las mujeres. $^{24}$

Con todo, a finales de los 70 ya se empezaban a plantear las implicaciones de la historia de las mujeres para la historiografía en general. En un artículo influyente dentro de la historiografía feminista, Joan Kelly argumentaba que el impacto de la historia de las mujeres giraba en torno a tres ejes principales: la periodización, las categorías de análisis, y las teorías de cambio social. ${ }^{25}$ El último de los elementos aludía a la relación entre propiedad privada y subordinación de la mujer; el segundo, referido a la situación de la mujer en las relaciones sociales entre los sexos, anticipaba los debates sobre el género que tendrían lugar a finales de los años 80 , que trataremos más adelante. Sin embargo, la cuestión de la periodización iba en línea con los elementos principales de la historia de las mujeres en los 70. Así, se argumentaba que la investigación del papel de las mujeres en diferentes procesos históricos, especialmente aquellos considerados como etapas de progreso - la Atenas clásica, el Renacimiento Italiano, o la Revolución Francesa-, ponía sobre la mesa que dichas etapas no habrían sido igualmente liberadoras. Así, la propia Joan Kelly, al analizar la situación de las mujeres en el Renacimiento Italiano, había observado que, con respecto a la Edad Media, las aristócratas habrían perdido opciones legales, políticas, sociales y económicas, un proceso que habría sido producto del surgimiento de los Estados modernos, una pujante economía comercial y una ideología que recluía a la mujer al espacio doméstico. ${ }^{26}$ En el fondo, este era el objetivo de la historia de las mujeres en los años 70: proporcionar nuevas interpretaciones a la luz de la consideración de las mujeres como sujeto colectivo, y valorar la situación histórica de las mujeres.

Esta breve explicación sirve como contexto intelectual para entender el estudio de la querella de las mujeres, ya que el desarrollo historiográfico sobre la querella ha estado marcado por el desarrollo del pensamiento feminista. Se han identificado tres

\footnotetext{
22 Joan Wallach Scott, Gender and the Politics of History (Columbia University Press: New York, 1999), $18-21$.

${ }^{23}$ M. Zancarini-Fournel, "Histoire des femmes", 209.

${ }^{24}$ Cristina Segura Graíño, "Cómo construimos la historia de las mujeres desde las universidades españolas", Revista de historiografía, 22 (2015): 262-267.

${ }^{25}$ Joan Kelly, "The social relations of the sexes: methodological implications of women's history", Signs, vol. 1, 4 (1976): 809-823.

${ }^{26}$ Joan Kelly, "Did women have a Renaissance?", en Renate Bridenthal, Claudia Koonz y Susan Stuard, Becoming visible: women in European history (Houghton Mifflin Company: Boston y Dallas, 1987), 175201.
} 
etapas en las cuales ha habido un esfuerzo historiográfico por analizar la controversia. En primer lugar, en la Francia de los años 30, se plantea el tema en el siglo XVI, buscando establecer los orígenes o antecedentes del feminismo en el debate. Se trataría de un intento de carácter historicista. Un segundo tiempo, en los años 60 y 70, posiblemente en relación con la creciente importancia de la historia de las mujeres, surge un nuevo interés por la querella. Se trata de un interés presente principalmente en Francia, aunque también en Italia. ${ }^{27}$ La concepción de la mujer como un sujeto histórico, produce un interés en el pensamiento de las mujeres del pasado, y en autoras de la querella como Christine de Pizan, por cuanto se interpreta que estas habrían identificado y criticado algunos aspectos de la subordinación femenina, caracterizando su pensamiento como una forma de "feminismo temprano". ${ }^{28}$ Una tercera etapa se daría a partir de los años 90, con un renovado interés por dicho fenómeno.

Con todo, las primeras aproximaciones historiográficas tienden a centrarse solo en algunos aspectos de este fenómeno. Tomaremos como ejemplo el artículo de Joan Kelly sobre la querella, que entiende las intervenciones femeninas en el debate como una suerte de feminismo temprano. En este se reflejan las preocupaciones que se han apuntado anteriormente:

a) Periodización y agencia femenina: un primer objetivo del texto de J. Kelly es reivindicar que la historia del feminismo no comienza en la Revolución Francesa, sino con Christine de Pizan (1364-1430). Si bien esta idea es anterior a la obra de J. Kelly, esta autora destaca que la literatura sobre el tema destacaba los discursos masculinos a favor de las mujeres, sin embargo se oscurecía la presencia de autoras como Mary Astell, Lucrezia Marinella y Aphra Behn. La autora problematiza, entonces, dos cuestiones. Por un lado, la periodización, en este caso, en relación a la historia del feminismo, que para J. Kelly tendría una primera fase, desde el siglo XV a finales del XVIII, que denomina feminismo temprano. ${ }^{29}$ Por otra, este feminismo temprano estaría caracterizado por la actividad intelectual de un grupo de mujeres que es consciente de su condición femenina, y critica la subordinación, aunque no desarrolla ninguna noción de progreso o cambio social.

b) Categorías de análisis: para J. Kelly, además, la distinción entre sexo y género sería fundamental para entender la querella. Así, las autoras que intervienen en la misma serían conscientes de la contingencia de ciertos fenómenos sociales, en particular el maltrato y la difamación del sexo femenino, que sería un fenómeno cultural. Así, por ejemplo, Christine de Pizan afirma haber interiorizado las continuas difamaciones contra las mujeres, antes de darse cuenta de que estas no representaban sus experiencias.

c) Cambio social: J. Kelly relaciona los discursos de estas mujeres con un conjunto de cambios sociales especialmente presentes a partir del siglo $\mathrm{XV}$, que para

\footnotetext{
${ }^{27}$ M. Zimmermann, "Querelle des femmes”, 85-87.

${ }^{28}$ Joan Kelly, "Early feminist theory and the 'Querelle des femmes', 1400-1789", Signs, vol. 8, 1 (1982): 4-28.

${ }^{29}$ En un comentario al artículo de J.Kelly, se ha destacado que "Kelly does not define her chronological period according to traditional epochs of historiography -the Middle Ages, the Renaissance and so forthbut creates her own era, 1400-1789, which supersedes the others”. S. Schibanoff, “Comment on Kelly's 'Early feminist theory and the querelle des femmes', 1400-1789”, Signs, vol. 9, 2 (1983): 320.
} 
ella consistirían en la construcción de los estados modernos, el humanismo, el surgimiento de un modo de vida burgués y un ethos republicano, que marcaría una división clara entre lo público y lo privado, de manera que se produce una pérdida del poder político, económico, social y cultural, que anteriormente detentarían ciertas mujeres aristócratas, apareciendo una nueva identidad o construcción de género, el de la "señora doméstica" relegada al plano privado. Así, las intervenciones en la querella serían discursos producidas por mujeres humanistas, que estarían protestando contra esta nueva realidad. ${ }^{30}$

De esta forma, el estudio de la querella de las mujeres refleja las líneas generales de la historiografía feminista en torno a la reflexión sobre la agencia femenina, la periodización, el cambio social, o las categorías de análisis. Ahora bien, el crecimiento de la historia de las mujeres, la sofisticación de los marcos teóricos o los cambios generales en la historiografía harán que, a partir de los años 80 , cambien los focos de interés a la hora de estudiar la querella.

\section{Querella de las mujeres: conciencia femenina, cuerpo y diferencia}

Desde finales de los años 70 comienzan a percibirse otras dinámicas que se harán patentes a lo largo de la década de los 80. En este sentido, la historia de las mujeres sigue los cambios acontecidos en la disciplina histórica, con el abandono progresivo de la historia social y económica en favor de una historia preocupada por los fenómenos culturales, con una fuerte presencia de elementos simbólicos. En Francia, por ejemplo, el paso de la historia de las mentalidades a modelos de carácter antropológico da lugar a nuevos objetos de estudios histórico que se incorporarán en el panorama más amplio de la historia de las mujeres: el cuerpo, los gestos, los rituales, las representaciones, el parentesco, los objetos materiales, o las diferentes culturas en una misma sociedad. ${ }^{31}$

Así, la historia de las mujeres pasa a estudiar temas como el parto, la maternidad, la salud y la belleza, así como los espacios cotidianos diferenciados entre mujeres y hombres. En suma, lo que se ha llamado cultura femenina, todo un entramado de símbolos propios de las mujeres en una sociedad dada. Esto supone una atención a nuevas fuentes: orales y materiales, archivos institucionales que permitan el acceso a información sobre el parto, archivos judiciales, pero también, documentos personales, fotografías o autobiografías. Es decir, todo material que permita indagar sobre los roles simbólicos asignados a los sexos, así como la información referida a la experiencia subjetiva de las mujeres. ${ }^{32}$ Además, la noción de "cultura femenina", con un espacio y actividades que son propios de mujeres, permitía plantearse la relación entre hombres y mujeres de una forma diferente. Las mujeres ya no eran exclusivamente víctimas de la dominación masculina, sino que podían aprovechar el entramado simbólico sobre el que se apoyaba dicha dominación. En el mundo rural francés del siglo XIX, los hombres no podían inmiscuirse en las tareas domésticas, una actividad considerada femenina, sin sufrir una desvalorización. ${ }^{33}$

\footnotetext{
${ }^{30}$ J. Kelly, "Early feminist theory", 5-8 y 13-14.

${ }^{31}$ Jacques Le Goff y Jacques Berlioz, “Anthropologie et histoire”, en Michel Balard (comp.), L'histoire médiévale en France. Bilan et perspectives (Éditions du Seuil: París, 1991), 267-304.

${ }^{32}$ M. Zancarini-Fournel, "Histoire des femmes", 210-212.

33 Arlette Farge, "Historia de las mujeres. Cultura y poder de las mujeres: ensayo de
} 
En línea con estos desarrollos, el marco teórico principal, a la hora de tratar la querella de las mujeres en el ámbito hispano, ha sido el llamado feminismo de la diferencia que aboga por estudiar a las mujeres por sí mismas, sin hacer referencia a los varones, centrándose en el pensamiento propio que desarrollan, que sería diferente a los discursos masculinos dominantes. ${ }^{34}$ Se trataría, en buena medida de una perspectiva influida por la historia de las mujeres practicada en Italia a partir de los años 80, si bien su recepción académica es escasa hasta la publicación, entre 1990 y 1992, de la obra colectiva Historia de las mujeres en Occidente, editada por Michelle Perrot y Georges Duby. ${ }^{35}$ Aunque no parece arriesgado situar esta perspectiva en la línea de las prácticas historiográficas de carácter antropológico de los años 80, el bagaje conceptual ha continuado aplicándose durante los años 90 y en el cambio de siglo, de forma que en la historia de las mujeres en España siguen teniendo un importante peso las representaciones, el acceso al poder, las formas de sociabilidad femenina o la educación de las mujeres; aspectos de peso en relación al concepto de "cultura femenina". ${ }^{36}$ Así, el fenómeno de la querella de las mujeres no ha sido ajeno a la teorización historiográfica en el ámbito castellano. Más bien, un grupo de autoras ha interpretado la controversia como la emergencia de una conciencia colectiva femenina que se rebela contra el patriarcado.

El feminismo de la diferencia funciona, a la hora de estudiar la querella, con un conjunto de conceptos extraídos de autoras feministas como Gayle Rubin, Luce Irigaray o la italiana Luisa Muraro. Juega un papel fundamental la noción del cuerpo femenino, que para estas autoras ha estado oscurecido por las representaciones de género, pero que sin embargo se puede entender como un conjunto de datos biológicos relevantes en un conjunto de relaciones sociales (matrimonio, maternidad) en las que las mujeres no son un sujeto activo sino pasivo. Este sería el núcleo central del patriarcado, el emplear en relaciones sociales los cuerpos de las mujeres, sin que estas tengan capacidad de decisión alguna en dichas relaciones sociales. ${ }^{37}$ En este sentido, algunas historiadoras han defendido que el patriarcado, es decir, el poder coactivo de los hombres, impide a las mujeres llevar a cabo actividades propias. La mujer en este esquema sólo importaría en un conjunto de relaciones sociales destinadas a la reproducción -sexo, matrimonio, maternidad-. Esta perspectiva ha llevado a argumentar que la querella de las mujeres es un movimiento intelectual colectivo, anclado en la toma de conciencia de una serie de mujeres (Christine de Pizan, Laura Cereta, Teresa de Cartagena, Nicolosa Sanuti) que, dándose cuenta de la situación opresiva que viven como mujeres, escriben discutiendo

historiografía", Historia social, 9 (1991): 81-83.

${ }^{34}$ Sobre las perspectivas a la hora de hacer historia de las mujeres en España, véase Cristina Segura Graíño, "Historia de las mujeres en la Edad Media", Medievalismo, 18 (2008): 260-267.

${ }^{35}$ Véase al respecto I. Morant, "Historia de las mujeres e historia", 295-296, dónde se destaca, por ejemplo, la influencia de la revista Memoria: rivista di storia delle donne, creada en 1980.

${ }^{36}$ Véanse los artículos de C. Segura Graíño, "Cómo construimos la historia", 255-271; e "Historia de las mujeres en la Edad Media”, 249-272.

37 "Si nos limitamos a entender el cuerpo de mujer como un conjunto de datos biológicos que entra en la historia a través de relaciones sociales definidas por los hombres del grupo [...] podremos decir efectivamente que el cuerpo femenino participa en los procesos generales de cambio social; pero no las mujeres. Porque no hay ahí sujeto activo de cambio social, sino únicamente pasivo u objeto [...]". María Milagros Rivera Garretas, "El cuerpo femenino y la querella de las mujeres (Corona de Aragón, siglo XV)", en Georges Duby y Michelle Perrot (dirs.), Christine Klapisch Zuber (dir.), Historia de las mujeres en Occidente, vol. 2, La Edad Media (Taurus: Barcelona, 1992), 594. 
las nociones tradicionales acerca de las capacidades de las mujeres para participar en la vida política, para pensar individualmente o para decidir sobre su cuerpo -a través del adorno- ${ }^{38}$

En efecto, podemos pensar la querella de las mujeres como fenómeno colectivo que se produce en varios espacios del occidente medieval en el siglo $\mathrm{XV}$, pero cabría añadir algunos matices:

a) Si bien la sociedad medieval inserta, tanto en el pensamiento como en la práctica, a las mujeres en un conjunto de relaciones sociales en las que estas tienen una fuerte dependencia de los hombres, esto no quiere decir que esta dependencia sea absoluta. El matrimonio, por ejemplo, no es considerado necesariamente como una relación social en el que la mujer esté totalmente subordinada al hombre. Christine de Pizan, por ejemplo, defiende el papel de la mujer en el matrimonio, siempre que haya, por las dos partes, amor y fidelidad. ${ }^{39}$

b) Christine de Pizan, Laura Cereta, Nicolosa Sanuti o Teresa de Cartagena no defienden que todas las mujeres participen en el ámbito público, o que todas las mujeres pueden decidir sobre su vida independientemente. Su defensa es una defensa de un conjunto de mujeres particular: las nobles, las virtuosas, las intelectuales. Además, también existen hombres que escriben a favor de las mujeres.

La identidad de género de estas autoras es, por supuesto, relevante a la hora de entender la querella de las mujeres. Ahora bien, hay que entender esta identidad en la pertenencia a un grupo social que valora otro tipo de identidades. Es decir, la defensa de la mujer no va desligada de la defensa de la virtud femenina, de la nobleza o de las capacidades intelectuales, debido a que estos son valores que forman parte de las maneras que tienen un conjunto de personas para interpretar el mundo, las acciones de los demás, y las relaciones sociales. En este conjunto de personas no están incluidas solo mujeres, sino que también varones, como muestra la obra de Juan Rodríguez del Padrón, Diego de Valera o Álvaro de Luna.

Otro componente teórico de la aplicación del feminismo de la diferencia al marco de la querella es el concepto de "des/autorización". Aun existiendo una conciencia colectiva femenina, como argumentan estas autoras, la participación en el debate sería una intervención en un orden simbólico con primacía de lo masculino, en la que las mujeres, debido a la intervención de la política sexual del patriarcado, no están autorizadas, prima facie, a intervenir. Sin embargo, es posible para ellas emplear una serie de estrategias de "autorización", entre las que se han identificado varias. Entre ellas encontramos la "captatio benevolentia", pero también el recurrir al pensamiento revelado. Incluso antes de la irrupción de la querella, autoras como Hildegarda de Bingen (1089-1179) habrían empleado dichas estrategias. ${ }^{40}$ En el siglo XV, sin embargo, las estrategias se diversifican. Christine de Pizan recurre a su propia experiencia como mujer, Nicolosa Sanuti recurre a ejemplos mitológicos e históricos de

\footnotetext{
${ }^{38}$ María Milagros Rivera Garretas, "La querella de las mujeres: una interpretación desde la diferencia sexual", Política y cultura, 6 (1996): 25-39.

${ }^{39}$ A. Vargas Martínez, "'La ciudad de las damas"”, 34-36.

${ }^{40}$ M. Cabré i Pairet, "Estrategias de des/autorización femenina", 80.
} 
mujeres que han intervenido en la vida pública, y Laura Cereta se define como astróloga para negar acusaciones de plagio que se habían hecho contra su obra. ${ }^{41}$ Estos serían ejemplos de estrategias que justificarían, de forma simbólica, la intervención de las mujeres en el ámbito intelectual.

Con todo, la idea de que dichas estrategias legitiman la intervención de todo el sexo femenino en el debate, no parece del todo clara. Por ejemplo, Christine de Pizan emplea otros discursos para justificar su intervención, relatos que no justifican su intervención como mujer, sino como mujer noble, virtuosa y educada. En su obra, la Ciudad de las damas, se le aparecen tres figuras alegóricas femeninas -la Razón, la Rectitud y la Justicia-, que la animan a construir una ciudad -simbólica- reservada únicamente a las damas nobles y virtuosas. ${ }^{42}$ Las tres figuras alegóricas funcionan como una estrategia de autorización: se está indicando que su defensa de las mujeres se basa en la razón, la rectitud y la justicia, de forma que el motivo por el que puede hablar en público es debido a que tiene estas tres virtudes. Así, el motivo por el que puede participar en un debate público son sus capacidades intelectuales (Razón), éticas (Rectitud) y sociales (Justicia), lo que implica que quienes no participen de dichas capacidades, no deberían intervenir en el ámbito público. Se presenta, así, una normatividad, que se ve reforzada cuando se introduce la ciudad de las damas como una ciudad a las que únicamente tienen acceso aquellas mujeres que sean nobles y virtuosas. Estos procedimientos, de carácter simbólico, nos indican que la defensa de las mujeres no es una defensa de todas las mujeres, sino de las mujeres que pertenecen a un grupo social determinado, que se guía por valores intelectuales, éticos y sociopolíticos. Así, parece conveniente plantear un marco teórico en el que se tengan en cuenta, desde luego, las diferencias sexuales y socioculturales entre hombres y mujeres, pero que, al mismo tiempo sitúe estas diferencias de género en relación a otras distinciones sociales. Es decir, es posible plantearse que los hombres y mujeres no son agentes masculinos y femeninos, en abstractos, sino que pertenecen también a clases sociales o comunidades, que mantienen uno u otro estatus, y participan de diferentes discursos. En efecto, una crítica de estas características a la historia de las mujeres, tal y como era practicada entre los años 70 y 80, es uno de los motivos que da lugar al desarrollo de la historia de género, especialmente a partir de los 90 .

\section{La historia de género y sus posibles consecuencias para la querella de las mujeres en Castilla}

Los años 90, entonces, suponen un momento de cambio en la manera de historiar las relaciones entre los sexos. Conceptos como el de "cultura femenina" se perciben ahora como inadecuados, debido a que suponen una suerte de naturalización de las diferencias entre los sexos, y son incapaces de dar cuenta de la relación entre los cambios económicos, sociales o políticos, y la vida cotidiana del sexo femenino. ${ }^{43}$ Las diferencias a la hora de hacer historia de las mujeres son especialmente patentes en los debates en torno a la publicación, entre 1990 y 1992, de los cinco tomos de Historia de las mujeres en Occidente, dirigidos por Michelle Perrot y Georges Duby. Se ponen de

\footnotetext{
${ }^{41}$ Dichas acusaciones, dentro del marco del feminismo de la diferencia, serían precisamente estrategias de desautorización propias de un orden simbólico masculino. Ibid., 82-98

${ }^{42}$ A. Vargas Martínez, "'La ciudad de las damas"”, 28-32.

${ }^{43}$ A. Farge, "Historia de las mujeres", 86.
} 
manifiesto algunas de las características de la historia de las mujeres en el ámbito internacional, especialmente la división entre las maneras de hacer historia de las mujeres en los países de habla anglosajona y la Europa continental. Por otra parte, destaca la influencia de los Annales y la antropología histórica que se venía realizando en Francia. De nuevo, la obra no estuvo exenta de críticas que se articulan en torno a varios ejes:

a) El propio objeto -las mujeres en la historia-, considerado aún prematuro, lo que podría llevar a la creación de un conjunto de temas y momentos privilegiados de forma precipitada.

b) El espacio, Occidente, considerado una categoría abstracta y globalizadora que, por una parte, subsume las diferencias regionales dentro del propio espacio -posibles diferencias entre Inglaterra y Francia, por ejemplo-, y, por otra, excluye otros territorios.

c) La cuestión metodológica, la pregunta de si se debe hacer historia de las mujeres, o bien una historia de las relaciones entre los sexos, lo que remite al concepto de género. La idea-fuerza sería que las categorías de "lo masculino" y "lo femenino" no son inamovibles, y que no se deben definir sólo en relación una con otra, sino también con lo excluido -androginia o travestismo, por ejemplo-.

d) En último lugar, se ha reprochado que se preste demasiada atención al discurso, es decir, que se construya la historia de las mujeres como una historia de las representaciones, en detrimento de la historia social y económica puesta en práctica en los años $70 .^{44}$

Estas críticas reflejan todo un conjunto de cuestiones que se estaban poniendo sobre la mesa a finales de los años 80 y en la primera mitad de los años 90 en relación al desarrollo del feminismo. No se trata únicamente de la emergencia del género como categoría analítica, sino también de las insuficiencias de la "segunda ola". El movimiento recibe críticas desde varios frentes. En primer lugar, una serie de autoras que consideran que el feminismo, aunque habiendo desempeñado un papel importante en la sociedad, se estaba convirtiendo en un movimiento desconectado de la sociedad, que ponía a las mujeres como colectivo en una situación de "victimización" y era incapaz de encontrar un lugar para la relación entre hombres y mujeres en la sociedad. Es lo que se denominó "posfeminismo". ${ }^{45}$ En segundo lugar, la emergencia de la "tercera ola" que critica al feminismo anterior la conceptualización del sexo femenino como un grupo homogéneo, de forma que no se explican la diferentes experiencias de las mujeres dependiendo de su raza, religión, región, o clase social. ${ }^{46}$ El propio concepto de "mujer" pasa a verse como una categoría inestable. ${ }^{47}$ Finalmente, la crítica a las categorías dicotómicas "hombre" y "mujer", que ocultarían y excluirían toda una serie

\footnotetext{
${ }^{44}$ Michelle Perrot, "Escribir la historia de las mujeres: una experiencia francesa", Ayer, 17 (1995): 78-81.

${ }^{45}$ Sarah Gamble, "Postfeminism", en Id. (ed.), The Routledge companion to feminism and postfeminism (Routledge: London, 2001), 43-47.

${ }^{46}$ E. Hernández Sandoica, Tendencias historiográficas actuales, 447-448.

${ }^{47}$ Kathleen Canning, Gender history in practice. Historical perspectives on bodies, class and citizenship (Cornell University Press: Ithaca y Londres, 2006), 8-9.
} 
de realidades de género que no se ubican fácilmente dentro de ellas -homosexualidad, androginia, travestismo o transexualidad, por ejemplo-; desarrollándose la llamada teoría "queer". 48

Todas estas críticas, tanto al feminismo de segunda ola en sus diversas variantes, como a la historia de las mujeres tal y como se venía practicando, apuntan a la imposibilidad de describir la experiencia de las mujeres como si estas pertenecieran a un colectivo unificado y homogéneo, afirmando que es necesario conceptualizar las diferencias de clase, raza, religión, área geográfica o cultura. ${ }^{49}$ Además, se considera poco adecuado estudiar la experiencia femenina sin tener en cuenta su relación con los varones. Se avanza entonces hacia un dominio historiográfico distinto, que gira entorno a la noción de género.

Aunque en un primer momento, el concepto de género surge como un concepto de análisis histórico dentro de la historia de las mujeres, el término se usa, especialmente desde finales de los 80, para designar realidades que no son abarcables desde la historia de las mujeres. La categoría de género se refiere fundamentalmente a dos aspectos sociales de la diferencia sexual. En primer lugar, la construcción cultural de lo masculino y lo femenino. ${ }^{50} \mathrm{El}$ concepto apunta, en este sentido, a los aspectos construidos social y culturalmente de las categorías de hombre y mujer, aspectos que pueden abarcar una mayor cantidad de instancias dependiendo de la perspectiva de análisis -feminismo radical, feminismo marxista, psicoanálisis, o posestructuralismo-. En segundo lugar, a las diferentes relaciones de poder que se derivan de dichas construcciones de lo femenino y lo masculino. Dentro del análisis del género, se supone que una sociedad dada naturaliza las categorías de lo masculino y lo femenino, una naturalización que legitima las relaciones de poder entre hombres y mujeres. ${ }^{51}$ Así, por ejemplo, en el mundo medieval la asociación del hombre con lo racional y de la mujer con lo pasional lleva como corolario que la mujer debe estar subordinada al hombre, algo que se plasma en el derecho canónico. ${ }^{52}$ En este sentido, la noción de género tiene también implicaciones de carácter político. El concepto va de la mano del desarrollo del feminismo en los años 80 y 90, con el objetivo de explorar las posibilidades de pensar las relaciones entre los sexos de forma distinta.

Sin embargo, la asociación más o menos generalizada, a nivel teórico, del género con estos dos elementos - construcción cultural y relaciones de poder- no significa que el uso de esta categoría haya estado exenta de controversia. Todo lo contrario. La

\footnotetext{
${ }^{48}$ Françoise Thébaud, "Género e historia en Francia: los usos de un término y de una categoría de análisis", Cuadernos de historia contemporánea, 28 (2006): 51-53.

${ }^{49}$ Destacan en este sentido los estudios estadounidenses en relación a la emergencia de identidades en la intersección entre raza, clase y género, lo que se conoce como interseccionalidad. Así, por ejemplo, se presenta la raza como un "metalenguaje" que afecta a las representaciones de clase y género. Véase K. Canning, Gender history in practice, 7-8; y Teresa A. Meade, y Merry E. Wiesner-Hanks, "Indroduction", en Id. (eds.), A companion to gender history (Blackwell: Oxford, 2004), 6.

${ }^{50}$ Cabe destacar, además, que esta construcción no es únicamente una representación, sino que el género sería algo que "se hace", es decir, tiene un componente performativo. Véase Candace West y Don H. Zimmerman, "Doing Gender", Gender \& Society, vol. 1, 2 (1987): 125-151. Es necesario destacar también la obra de la filósofa Judith Butler, especialmente influyente en la teoría "queer".

${ }^{51}$ E. Hernández Sandoica, Tendencias historiográficas actuales, 442-443 y 451-452.

52 Carmen Pallares Méndez, Historia das mulleres en Galicia. Idade Media (Nigratrea: Santiago de Compostela, 2011), 118-127.
} 
extensión de su uso en los años 80, se debía mucho más al carácter aparentemente más neutro del concepto que a una reflexión teórica sobre las implicaciones analíticas de su uso. Así, el género se empleaba a menudo para designar el estudio descriptivo de las mujeres, o de las relaciones entre los sexos en un momento dado. El término permitía, en este sentido, alejarse de la historia de las mujeres practicada en el momento, que se entendía como una forma de hacer historia propia de un feminismo militante. ${ }^{53}$ Además, la distinción entre sexo y género, es decir, entre los aspectos más o menos estables de la diferencia sexual -el sexo-, y lo construido culturalmente -el género-, no resultaba cómodo para algunas historiadoras, ya que limitaba las capacidades explicativas del concepto y al mismo tiempo suponía aceptar que existen diferencias inmutables entre hombres y mujeres. ${ }^{54}$ Posiblemente, quien ha ejercido más influencia en la disciplina histórica a la hora de criticar cualquier tipo de perspectiva que naturalice las diferencias entre hombre y mujer ha sido Joan Scott. Adoptando perspectivas posestructuralistas, en un artículo de 1986, esta autora criticaba los enfoques del feminismo radical, del feminismo marxista, y las perspectivas psicoanalíticas a la hora de dar cuenta de las diferencias entre los géneros. ${ }^{55}$ Para la autora, todo lo relativo a lo masculino y lo femenino son construcciones culturales, significados que se imponen en las diferencias entre los sexos a través del lenguaje. ${ }^{56}$ De hecho la propia noción de diferencias entre los sexos serían, al menos parcialmente, un producto del lenguaje.

El género supone, entonces, una perspectiva relacional. El término expresa las representaciones y prácticas producidas socialmente que llevan a diferentes tipos de relaciones, entre varones y mujeres o entre miembros de un mismo sexo. Así, aunque la historia de la mujeres pueda hacer uso del concepto de género, hay una serie de cuestiones -las representaciones de los enemigos como "afeminados" en el ámbito bélico, por ejemplo- que trasciende a la historia de las mujeres, abriendo un nuevo campo que corresponde, más bien, a la historia de género.

El concepto de género, tal y como lo hemos desarrollado aquí, tiene consecuencias relevantes para el estudio de la querella de las mujeres, ${ }^{57}$ y en general para el estudio de la Edad Media. ${ }^{58}$ En el caso de la querella de las mujeres, supone no

\footnotetext{
${ }^{53}$ J. Wallach Scott, Gender and the Politics of History, 31-32.

54 Gisela Bock, "La historia de las mujeres y la historia del género: aspectos de un debate internacional”, Historia social, 9 (1991): 68-69. Esta autora está especialmente preocupada por que la distinción entre sexo y género lleve a debates estériles sobre los aspectos de lo femenino y lo masculino se deben atribuir a cada ámbito, así como por los peligros que supondría un pensamiento "biologicista".

55 Joan Wallach Scott, "Gender: a useful category for historical analysis", American Historical Review, vol. 91, 5 (1986): 1053-1075. Para Scott, el género es "a constitutive element of social relationships based on perceived differences between the sexes, and gender is a primary way of signifying relationships of power" (p. 1067).

${ }_{56}$ J. Wallach Scott, Gender and the Politics of History, 33-44.

57 Mónica Bolufer y Monserrat Cabré, "La Querelle des femmes en Espagne: bilan sur 1'histoire d'un débat (1400-1800)", en Marie-Elisabeth Hennau y Rotraud von Kulessa (eds.), Revisiter la querelle des femmes. Discours sur l'égalitélinégalité des femmes et des hommes à l'échelle européenne (Presses universitaires de Saint-Étienne: Saint-Étienne, 2015), 44.

${ }^{58}$ La bibliografía sobre género en la Edad Media es extensa. Una pequeña muestra: Caroline Bynum, Holy feast and holy fast: the religious significance of food for medieval women (University of California Press: Berkeley, 1987); Joan Cadden, The meanings of sex difference in the Middle Ages: medicine, science and culture (Cambridge University Press: Cambridge, 1993); Juddith Bennett, "Medievalism and feminism", Speculum, 68 (1993): 309-331; Jo Ann Mcnamara, "The herrenfrage: the reestructuring of the gender system, 1050-1150”, en Clare A. Lees (ed.), Medieval masculinities: regarding men in the Middle
} 
asumir que las defensas (o las críticas) al sexo femenino reflejan la emergencia de una conciencia colectiva que abarca a todas las mujeres, o la mayor parte de ellas. Así, resulta conveniente estudiar a las autoras y autores de la querella como miembros de sectores sociales específicos, compuestos por varias identidades. Veamos algunas características del caso castellano.

En primer lugar, las defensas de mujeres parecen presentarse en buena medida contra obras de consideradas "difamantes" contra las mujeres. Tanto Rodríguez del Padrón como Diego de Valera se refieren a Giovanni Boccacio, autor de Il Corbaccio, una obra de este estilo, por ejemplo; y la querella parece desarrollarse en respuesta a la obra de Alfonso Martínez de Toleda, muy crítico con las mujeres, pero también crítico con los ideales del amor cortés. Este carácter polémico es un elemento central, que trataremos en este apartado, así como la idea de que la defensa no es una defensa de las mujeres en general, sino más bien de las integradas en grupo social determinado. Así, analizaremos algunas de las características de las representaciones de género que se producen en este marco.

Las representaciones de un grupo social a menudo se producen en contraposición con otro grupo social, real o imaginario. En este sentido, el uso de las representaciones funciona a menudo como una manera de distinción, de diferenciación. La querella de las mujeres en Castilla no es ajeno a este fenómeno, y se podría considerar que su carácter polémico viene dado, en buena medida, por esta cuestión. Así, todos los autores presentan sus obras como respuestas a los ataques de "maldizientes" y "difamadores". Juan de Mena, por ejemplo, que prologa el libro de Álvaro de Luna, indica que la obra se dirige a reprender a aquellos que "mancillaban" y "ofendían" a las "claras mujeres" de su época. ${ }^{59}$ Diego de Valera se propone atacar a la "secta" de "maldizientes" y defender la fama de las mujeres. ${ }^{60}$ Existe una preocupación por las difamaciones y un intento de diferenciación de aquellos que critican a las mujeres, a los que se caracteriza con términos como "maldizientes". En este sentido, entendemos que esto obedece a una cuestión social, la de oponer el grupo social propio a otros.

En buena medida, la oposición a los difamadores se da mediante otra representación, una interpretación del relato bíblico de la Caída, interpretación que reivindica la figura de Eva. Tanto Rodríguez del Padrón como Álvaro de Luna intentan exculpar a Eva. Argumentan, por ejemplo, que mientras que Adán fue creado a partir del barro y fuera del Paraíso, Eva fue creada a partir de la carne de Adán y dentro del paraíso, lo que la haría superior. Asimismo, Rodríguez del Padrón argumenta que es a Adán a quien se le había prohibido comer del fruto prohibido, no a Eva. ${ }^{61}$ Se trata de

Ages (University of Minnesota Press: Minneapolis, 1994), 3-30; Dyan Elliott, Fallen Bodies: pollution, sexuality and demonology in the Middle Ages (University of Pennsylvania Press: Philadelphia, 1998). Para algunas reflexiones recientes, véase Judith M. Bennettt y Ruth Mazo Karras, "Women, gender and medieval historians" en Id. (eds.), The Oxford handbook of women and gender in medieval Europe (Oxford University Press: Oxford, 2013), 1-17; y Dyan Elliott, "The three ages of Joan Scott", American Historical Review, vol. 113, 5 (2008): 1390-1403.

${ }^{59}$ M. J. Fuentes Pérez, "Voces profemeninas", 109-111.

${ }^{60}$ Florence Serrano, "Del debate a la propaganda política mediante la querella de las mujeres en Juan Rodríguez de Padrón, Diego de Valera, y Álvaro de Luna”, Talia dixit: revista interdisciplinar de retórica e historiografía, 7 (2012): 103-106.

${ }^{61}$ M. J. Fuentes Pérez, "Voces profemeninas", 111-112; y A. Vargas Martínez, "Sobre los discursos 
una interpretación del Génesis que supone una representación del género, de las relaciones entre el hombre y la mujer, diferenciado de la interpretación dominante. La crítica a la interpretación tradicional del Génesis es, posiblemente, fruto de un intento de diferenciación de los defensores de mujeres y el grupo social que representan, en contraposición con las difamaciones, en las que la culpabilidad de Eva jugaría un papel central. $^{62}$

Con todo, el aspecto de género más relevante es la construcción de un ideal de mujer virtuosa, noble e intelectual o educada. Se trata de una representación que refleja las aspiraciones de los hombres y mujeres de la Corte -0 , más bien, de un sector de la misma-. No es que todas las mujeres de este grupo social fueran virtuosas e intelectuales, pero sí que se trata de ideales que guían el comportamiento de hombres y mujeres, que representan un comportamiento a aspirar, así como aspectos de la forma de entender el mundo de este colectivo.

Una de las características fundamentales de este ideal sería la virtud. La defensa que hace Álvaro de Luna, a través de un catálogo de mujeres ilustres de la Biblia, de la Antiguiedad y de la Cristiandad es una defensa de mujeres que se comportan de acuerdo con una serie de estándares, mujeres que representan virtudes como la castidad, la obediencia, la prudencia o la honestidad. El uso del término virtud, una cuestión ética, pronuncia aún más el carácter normativo de este ideal de mujer, un ideal a seguir por las mujeres. Además, las mujeres que acceden al espacio público, las que desempeñan un rol político son mujeres con virtudes masculinas, es decir, viragos. ${ }^{63}$ El tipo de mujer que interviene en política, es un tipo de mujer poco común, poco femenino, un ideal de mujer diferente y no alcanzable para la mayoría. No se trata de atacar al sistema de género, sino de poner sobre la mesa una serie de representaciones que destacan las cualidades positivas de la mujer, representaciones que usa parte de la sociedad cortesana para interpretar y dar sentido a su realidad social.

La virtud no es el único elemento destacado sino también la nobleza. En este sentido, resulta claro el caso de Rodríguez del Padrón. El Triunfo de las donas está escrito como una suerte de prólogo a la Cádira de Honor, una obra sobre la nobleza, y el autor se pregunta, en el Triunfo, a quién debe dedicar la Cádira llegando a la conclusión de que debe hacerlo a la persona más noble, María de Aragón. La defensa de las mujeres es una defensa de las mujeres nobles, cualidad que está relacionada con la virtud. Por otra parte, se ha querido ver en la defensa del adorno por parte de Rodríguez del Padrón un elemento subversivo, contrario a la opinión generalizada, ya que hasta en las defensas de mujeres se critica el uso excesivo del adorno. ${ }^{64}$ Sin embargo, también podría verse como producto de la condición del autor como poeta de amor cortés, en el que la idealización de la dama jugaba un papel esencial. Así, la defensa del adorno en relación a la belleza femenina que hace Rodríguez del Padrón respondería a otras adscripciones sociales del autor, no necesariamente a un intento de atacar las relaciones de género establecidas.

políticos", 280 .

${ }^{62}$ Sobre esta interpretación véase R. Howard Bloch, Medieval misoginy and the invention of western romantic love (The University of Chicago Press: Chicago \& London, 1991), 22-26.

${ }^{63}$ M. J. Fuentes Pérez, "Voces profemeninas", 111-116.

${ }^{64}$ A. Vargas Martínez, "Sobre los discursos políticos", 282. 
Un ejemplo de la relevancia del ámbito intelectual en la construcción de estos ideales son las representaciones de Teresa de Cartagena en Admiraçión operum Dey. Aunque esta autora presenta ciertas diferencias con los anteriores -escribe en la segunda mitad del siglo XV, es religiosa, no está directamente relacionada con la corte y escribe en respuesta a las críticas a su anterior obra- es posible encuadrarla dentro de la querella. El elemento más relevante de su discurso sería la capacidad que tienen las mujeres, como los hombres, de acceder al conocimiento. Y esta posibilidad viene dada, para la autora, por la posibilidad de Dios de conceder bienes de la gracia a cualquier persona. Si bien se presenta a hombres y mujeres con cierta igualdad en este punto, también se los diferencia en cuanto a su acceso a la fuerza, al poder y a la riqueza. Lo que se está admitiendo en última instancia es la capacidad de las mujeres para participar en los discursos públicos, en la actividad cultural e intelectual. Y es posible relacionar esto con la sociedad cortesana. Teresa de Cartagena escribe Admiraçión operum Dey como respuesta a las críticas a su primera obra, Arboleda de los enfermos, que criticaban que una mujer pudiera escribir un texto así. La respuesta viene promovida, en parte, por Juana de Mendoza, una mujer de la corte, lo que indica la relevancia que tendría la defensa de las capacidades intelectuales de la mujer en la sociedad cortesana. ${ }^{65}$ De nuevo, el discurso de Teresa de Cartagena representa en buena medida los ideales, valores y categorías de la corte, y este discurso es una muestra de un proceso de diferenciación de un grupo cortesano.

Estudiar la querella desde la historia de género, no supone únicamente poner las representaciones de las mujeres en relación a los grupos sociales en los que estas se construyen. Para la historia de género lo femenino no es la única construcción social y cultural relevante a la hora de estudiar las relaciones entre los sexos. Más bien, supone revelar que la noción de masculinidad tienen tanto de construido como lo femenino. Así, la aplicación sistemática de la noción de género lleva a estudiar las representaciones sociales de los hombres, los roles asignados a los mismos o la construcción de la identidad masculina así como las crisis que esta puede sufrir. La ausencia de la diferencia sexual en la historia tradicional cobra, entonces, otro significado. Si bien antes de la emergencia de la historia de las mujeres, la historia era en, muchos sentidos, una historia de los hombres, no se tenía en cuenta esta identidad de género. Sin embargo, la noción de género apunta a la posibilidad de hacer una historia de los hombres en cuanto hombres. Se trata de estudiar aquí la importancia de lo bélico, de lo deportivo o de lo académico a la hora de formar las representaciones sobre lo masculino, así como las diferentes formas de entender la masculinidad dependiendo de la clase social, la raza o la religión. ${ }^{66}$

Efectivamente, este ha sido uno de los ámbitos de estudio más relevantes entre los usos del género como herramienta analítica que se han dado desde los años 90. La mayor abundancia de fuentes referentes a hombres así como la novedad de esta disciplina han hecho de la historia de las masculinidades un campo fundamental de estudio histórico del género. Incluso los discursos sobre mujeres realizados por hombres, cuyo uso excesivo había sido criticado por centrarse en las representaciones y

\footnotetext{
${ }^{65}$ M.-M. Rivera Garretas, "La Admiración de las obras de Dios", 288-289.

${ }^{66}$ Algunos ejemplos en Joanne Meyerowitz, "A History of 'Gender”, American Historical Review, vol. 113, 5 (2008): 1348-1353.
} 
no en las prácticas que estas producían, pasan a ser una fuente que puede desvelar cuestiones sobre la construcción de la masculinidad o incluso sobre las crisis de identidad masculina. ${ }^{67}$ Ahora bien, esta práctica no ha estado exenta de críticas, especialmente desde la historia de las mujeres. La multiplicación de trabajos sobre la historia de los hombres ha sido vista como una amenaza, ya que contribuiría a ocultar de nuevo la posición de las mujeres como sujetos históricos. ${ }^{68}$

El caso de la querella de las mujeres en Castilla puede ser un buen espacio para reflexionar sobre la construcción de las masculinidades sin dejar de lado la referencia al sexo femenino. La querella gira entorno a la representación de las mujeres, desde un determinado colectivo -que incluye como rasgo principal la vinculación a la corte de la mayoría de los miembros-, y, además, la mayor parte de las intervenciones, al menos durante el reinado de Juan II, son discursos realizados por varones. Así, estos textos pueden servir para reflexionar sobre las formas en las que los hombres cortesanos pensaban su relación con las mujeres y, por extensión, su propia masculinidad. En este sentido, se puede indicar como posibilidad que autores como Juan Rodríguez del Padrón, Diego de Valera o Álvaro de Luna, no hacen más que reivindicar los valores cortesanos en auge en el siglo XV, en especial en relación a la "divinización" -al menos lírica- del amor y de la dama. ${ }^{69} \mathrm{Y}$ esta reivindicación se haría precisamente, frente a críticas -"difamaciones"- como las del Corbacho, que atacan al amante cortés como un modelo masculino negativo.

\section{Conclusiones}

A lo largo de este artículo hemos visto cómo la evolución del estudio de la querella de las mujeres, especialmente en Castilla, ha estado marcado por el desarrollo general de la historia de las mujeres y la historia de género. Las primeras investigaciones, como se percibe en el artículo de Joan Kelly, se preocupan destacar las intervenciones femeninas de la querella como un fenómeno propio de la agencia femenina, y de utilizar dichas intervenciones para construir nuevas periodizaciones -el caso del "feminismo temprano"-, u otras formas de entender el cambio social. Así mismo, desde el feminismo de la diferencia, que comparte algunas características con la historia de las mujeres practicada en los años 80, con su énfasis en la cultura femenina, se ha construido un aparato conceptual -el cuerpo femenino, el patriarcado, la agencia y la conciencia femenina, las estrategias de des/autorización- que permite interpretar la querella como la emergencia de una conciencia colectiva femenina, de la que autoras como Christine de Pizan o Teresa de Cartagena una suerte de portavoces.

Sin pretender desacreditar estas perspectivas, nos parece que el estudio de la

\footnotetext{
${ }^{67}$ Gisela Bock, en efecto, considera que el pensamiento sobre las mujeres, incluido tradicionalmente dentro de la historia de las mujeres, debería encuadrarse más bien estudios sobre los hombres. G. Bock, 'La historia de las mujeres", 72-73.

${ }^{68}$ Kathryn Geadle, “The imagined communities of women's history: current debates and emerging themes, a rhizomatic approach", Women's history review, vol. 22, 4 (2013): 535-540

69 Véase al respecto E. Michael Gerli, "La 'Religión del Amor' y el antifeminismo en las letras castellanas del siglo XV”, Hispanic Review, vol. 49, 1 (1981): 65-86.
} 
querella puede hacerse también desde la historia de género, entendida esta como una historia de la construcción de lo masculino y lo femenino en relación a otras diferencias sociales. Así, se han indicado algunos aspectos que se pueden tener en cuenta a la hora de construir una historia de este tipo. Por una parte, a pesar de los diferentes tiempos y espacios de la querella en el siglo XV, se han destacado algunos rasgos comunes como el carácter polémico de los textos, o las adscripciones sociales cortesanas de los autores y autoras -al menos en los casos castellano y francés-. Además, es posible hacer alguna generalización en relación a las representaciones que emplean las defensas de las mujeres -la importancia de la virtud, la figura de Eva, el recurrir a mujeres ejemplares-. Este fenómeno, según nos parece, obedece a una dinámica colectiva de las representaciones, en la que estos discursos son reflejo de los valores e ideales del ámbito de la Corte. De este modo las defensas de las mujeres no son, principalmente, defensas de todas las mujeres, sin tener en cuenta su estatus social, sino que se defiende a las mujeres de la Corte, mujeres nobles, consideradas virtuosas, cuya idealización jugaría un papel importante en la construcción de un tipo de masculinidad específico, el del amante cortés.

\section{Profile}

Roque Sampedro es graduado en Historia por la Universidad de Santiago de Compostela (2014) y Máster en Estudios Medievales en la misma universidad (2017). Actualmente se encuentra realizando estudios de doctorado en la Universidad de Santiago de Compostela.

Roque Sampedro graduated in history at the Universidad de Santiago de Compostela (2014) and holds a Máster's degree in Medieval Studies at the same university (2017). $\mathrm{He}$ is now pursuing a $\mathrm{PhD}$ in Medieval Studies.

Fecha de recepción: 5 de febrero de 2018.

Fecha de aceptación: 30 de julio de 2018.

Publicación: 31 de diciembre de 2018.

Para citar este artículo: Roque Sampedro, "La querella de las mujeres en Castilla (siglo $\mathrm{XV}$ ) y su relación con la historia de las mujeres y la historia de género", Historiografías, 16 (julio-diciembre, 2018): pp. 36-56. 